\title{
Outbreak by Ventilator-Associated ST11 K. pneumoniae with Co-production of CTX-M-24 and KPC-2 in a SICU of a Tertiary Teaching Hospital in Central China
}

\section{OPEN ACCESS}

Edited by:

Octavio Luiz Franco,

Universidade Católica de Brasilia,

Brazil

Reviewed by:

Alex Leite Pereira,

University of Brasilia, Brazil

Carina Elisei Oliveira,

Universidade Católica Dom Bosco,

Brazil

*Correspondence:

Fangyou Yu

wzjxyfy@163.com

Liangxing Wang

38805@163.com

tThese authors have contributed equally to this work.

Specialty section:

This article was submitted to

Antimicrobials, Resistance

and Chemotherapy,

a section of the journal

Frontiers in Microbiology

Received: 03 April 2016

Accepted: 19 July 2016

Published: 02 August 2016

Citation:

Hu L, Liu Y, Deng L, Zhong Q, Hang $Y$, Wang $Z$, Zhan $L$, Wang $L$

and Yu F (2016) Outbreak by

Ventilator-Associated ST11

K. pneumoniae with Co-production

of CTX-M-24 and KPC-2 in a SICU

of a Tertiary Teaching Hospital

in Central China.

Front. Microbiol. 7:1190.

doi: 10.3389/fmicb.2016.01190

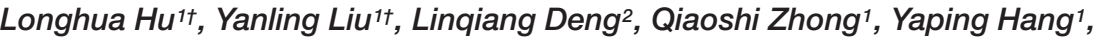 Zengzeng Wang ${ }^{3}$, Lingling Zhan $^{3}$, Liangxing Wang ${ }^{4 *}$ and Fangyou $\mathrm{Yu}^{3 *}$}

${ }^{1}$ Department of Clinical Laboratory, The Second Affiliated Hospital of Nanchang University, Nanchang, China, ${ }^{2}$ Department of Clinical Laboratory, Jiangxi Provincial People's Hospital, Nanchang, China, ${ }^{3}$ Department of Laboratory Medicine, The First Affiliated Hospital of Wenzhou Medical University, Wenzhou, China, ${ }^{4}$ Department of Respiratory Medicine, The First Affiliated Hospital of Wenzhou Medical University, Wenzhou, China

The emergence of carbapenem-resistant Klebsiella pneumoniae (CRKP) often responsible for numerous hospital-associated outbreaks has become an important public health problem. From January 2013 to February 2014, a total of 41 nonduplicate $K$. pneumoniae isolates with carbapenem resistance, were collected at a tertiary teaching hospital in Nanchang, central China. Among $41 \mathrm{~K}$. pneumoniae isolates, 28 were isolated from hospitalized patients including 19 from the patients in surgery intensive care unit (SICU) and 13 were isolated from ventilators. Twentyfour of 28 patients infected by CRKP have been submitted to mechanical ventilation using ventilator. More than 95\% of the CRKP isolates were resistant to 13 antimicrobials tested. All CRKP isolates were confirmed as carbapenemase producer and were positive for bla $a_{K P C-2}$, with one positive for both bla $a_{K P C-2}$ and $b / a_{N D M-1}$. All carbapenemase-producing isolates harbored at least one of extended spectrum $\beta$-lactamase genes tested, among which $95.1 \%$ (39/41) of the tested isolates were found to harbor both bla $\mathrm{C}_{C T}-M-24$ and $b_{1} a_{K P C-2}$, Of note, one isolate harbored simultaneously two carbapenemase genes (b/a $\mathrm{K}_{K P C-2}$ and bla $\left.\mathrm{NDM}_{-1}\right)$ and two ESBL genes (bla $C T X-M-3$ and bla $\left.a_{T E M-104}\right)$. To the best of our knowledge, coexistence of bla $K P C-2$ and bla $C T X-M-24$ in one isolate is first reported. MLST results showed that 41 CRKP isolates belonged to four sequence types (STs) including ST11, novel ST1854, novel ST1855, and ST1224. PFGE results displayed three PFGE clusters. Thirtyeight ST11 CRKP isolates $(92.7 \%, 38 / 41)$ including all 13 isolates from ventilators and 25 isolates from patients from seven wards (18 from SICU) belonged to same PFGE cluster, indicating these isolates were clonally related. Fifteen isolates have an identical undistinguished pattern (100\% similarity) forming a single clonal population. Moreover, this clone was exclusively linked to the cases attended in SICU and linked to the Ventilators. Additionally, the other SICU cases were linked to closely related 
clones (similarity greater than 95\%). These data indicated that the occurrence of a clonal outbreak associated with ventilators has been found. In conclusion, outbreak by ventilator-associated ST11 K. pneumoniae with co-production of CTX-M-24 and KPC-2 is found in a SICU of a tertiary teaching hospital in central China.

Keywords: K. pneumoniae, carbapenemase, KPC-2, outbreak, ST11

\section{INTRODUCTION}

Klebsiella pneumoniae is an opportunistic pathogen associated mainly with pneumonia, bloodstream infections, and urinary tract infections. K. pneumoniae frequently exhibits multiresistance to antimicrobial agents (Bratu et al., 2005). Carbapenems are often used for the treatment of clinical infections caused by this clinically important pathogen with multi-resistance to antimicrobial agents. However, the resistance of K. pneumoniae to carbapenems has been increasing (Gupta et al., 2011). As frequently carry additional antimicrobial resistance genes, $K$. pneumoniae isolates with carbapenem resistance often exhibit resistance to other non- $\beta$-lactam antimicrobial agents (Temkin et al., 2014). The emergence of carbapenem-resistant $K$. pneumoniae has become an important public health problem. The mechanism of resistance to carbapenems is mainly associated with production of carbapenemases (Nordmann and Poirel, 2002). Acquired carbapenemases encoded by transferable genes located in mobile elements including plasmids and transposons disseminate rapidly among different strains and species among Enterobacteriaceae (Temkin et al., 2014). K. pneumoniae carbapenemases (KPCs) and class B metallo- $\beta$-lactamases (MBLs) are found to be main carbapenemases in K. pneumoniae. In China, KPC-2 is a predominant carbapenemase and has been widely recognized in many species of Enterobacteriaceae including K. pneumoniae, Serratia marcescens, Escherichia coli, and Enterobacter cloacae (Cai et al., 2008; Wu et al., 2010). In 2009, a novel MBL, New Delhi metallo-lactamase 1 (NDM-1) initially identified in a $K$. pneumoniae isolate represents a new challenge for the treatment of infectious diseases (Yong et al., 2009; Kumarasamy et al., 2010). Dissemination of KPC-2 producing $K$. pneumoniae has been found in many Chinese hospitals (Yang et al., 2013; Hu et al., 2014). Recent reports showed that sequence type 258 (ST258) is the most prevalent clone contributing to the worldwide spread of KPC-producing K. pneumoniae and is the cause of numerous hospital-associated outbreaks (Kitchel et al., 2009; Hammerum et al., 2010; Marquez et al., 2014). In China, the dominant clone of KPC-producing K. pneumoniae is ST11 which is closely related to ST258 (Qi et al., 2011). Although many reports have identified KPC-2 producing $K$. pneumoniae, outbreak by ventilator-associated KPC-2 producing $K$. pneumoniae in China was limited. The aim of the present study was to investigate the dissemination of ventilator-associated $K$. pneumoniae with carbapenem resistance in a tertiary teaching hospital in central China. We demonstrated a outbreak by ventilator-associated ST11 K. pneumoniae with co-production of CTX-M-24 and KPC-2.

\section{MATERIALS AND METHODS}

\section{Isolation and Identification of Bacterial Strains}

From January 2013 to February 2014, a total of 41 nonduplicate $K$. pneumoniae isolates with carbapenem resistance were collected, among which 28 were isolated from hospitalized patients and 13 were isolated from ventilators in a tertiary teaching hospital in Nanchang, China. The 28 isolates of CRKP were isolated from sputum (22), blood (2), ascites (1), bile (1), urine (1), and bronchoalveolar lavage fluid (1) of the patients. The first isolation of every patient was selected for further investigation. Among 28 patients infected by CRKP, 19, 3, and 2 were isolated from surgical intensive care unit (SICU), respiratory ward and gastroenterology ward, respectively. Pulmonary infection was found among 22 of 28 patients, while bloodstream infection was found in two patients. The remaining four isolates were isolated from four patients in general surgery ward, neurosurgery ward, urology ward, and nephrology ward, respectively. Twenty-four of 28 patients have been submitted to mechanical ventilation using ventilator. Consequently, 13 CRKP isolates were isolated from the ventilators used for the patients mentioned above. The patients were primarily elderly (median age of 62.5 years old, average of 23-97 years old) and subjected to multiple underlying diseases. Twenty-six of 28 patients have been submitted to multiple invasive treatment procedures, such as mechanical ventilation (24/26), venipuncture and catheterization $(17 / 26)$, urinary catheterization (12/26). Moreover, all patients received multiple antimicrobials treatment in the course of hospitalization. Ventilators were sampled by rotating sterile, cotton-tipped swabs inserted into the bottom of the tubes. The bacterial isolates from sputum specimens growing more than three fourths of the plate by quantitative culture were considered to be responsible for pulmonary infection. K. pneumoniae isolates were identified by a Vitek-2 microbiology analyzer (bioMérieux, Marcy l'Etoile, France) in accordance with the manufacturer's instructions. Escherichia coli ATCC 25922 was used as the control strain. The Ethics Committee of the first Affiliated Hospital of Wenzhou Medical University exempted this study from review because the present study focused on the bacteria.

\section{Antimicrobial Susceptibility Testing}

Antimicrobial susceptibilities were determined initially by using Gram-negative susceptibility (GNS) cards on the Vitek system (bioMérieux, Marcy l'Etoile, France). Then multidrug resistance profiles were further evaluated by the disk diffusion test using commercial disks including minocycline $(30 \mu \mathrm{g})$, tetracycline $(30 \mu \mathrm{g})$, piperacillin $(100 \mu \mathrm{g})$, piperacillin/sulbactam 
(110 $\mu \mathrm{g})$, cefotaxime $(30 \mu \mathrm{g})$, ceftazidime (30 $\mu \mathrm{g})$, cefepime (30 $\mu \mathrm{g})$, aztreonam $(30 \mu \mathrm{g})$, cefoxitin $(30 \mu \mathrm{g})$, imipenem (10 $\mu \mathrm{g})$, meropenem $(10 \mu \mathrm{g})$, trimethoprim/sulfamethoxazole $(1.25 / 23.75 \mu \mathrm{g})$, amikacin $(30 \mu \mathrm{g})$, gentamicin $(10 \mu \mathrm{g})$, ciprofloxacin $(5 \mu \mathrm{g})$, and levofloxacin $(5 \mu \mathrm{g})$, in accordance with the criteria recommended by Clinical and Laboratory Standards Institute [CLSI] (2014). Escherichia coli ATCC 25922 was used as quality control strain for antimicrobial susceptibility testing.

\section{Detection of $\beta$-Lactamases}

The carbapenemases produced by CRKP isolates were determined by modified Hodge test recommended by Clinical and Laboratory Standards Institute [CLSI] (2014). Extended spectrum beta lactamases (ESBLs) were tested among all CRKP isolates by the CLSI-recommended confirmatory double disk combination test (Clinical and Laboratory Standards Institute [CLSI], 2014).

\section{Investigation of Resistance Genes}

The carbapenemase genes responsible for carbapenem resistance, including bla $a_{K P C}, b l a_{G E S}, b l a_{S P M}$, bla $a_{I M P}$, bla $a_{\mathrm{VIM}}, b l a_{S P M}$, and $b l a_{N D M}$, were detected using PCR and DNA sequencing as described previously (Queenan and Bush, 2007; Nordmann et al., 2011). ESBLs genes were detected in accordance with the method described previously (Andrade et al., 2010). PCR products were analyzed by electrophoresis in $1 \%$ agarose gels and were sequenced on both strands.

\section{Bacterial Clonal Relatedness}

Pulsed-field gel electrophoresis (PFGE) was established using $\mathrm{XbaI}$ digestion for $4 \mathrm{~h}$ at $37^{\circ} \mathrm{C}$ and electrophoresis in agarose gels for $19 \mathrm{~h}$ at $14^{\circ} \mathrm{C}, 120^{\circ}$, with switch times of 6 and $36 \mathrm{~s}$ at $6 \mathrm{~V} / \mathrm{cm}$, Bio-Rad CHEF III system. Comparison of the PFGE patterns was performed with Bionumerics software (Applied Maths, SintMartens-Latem, Belgium) using the Dice Similarity coefficient. Clusters were defined as DNA patterns sharing more than $80 \%$ similarity. Multi-locus sequence typing (MLST) was performed with seven standard housekeeping loci (gapA, infB, $m d h$, pgi, phoE, rpoB, and ton $B$ ) as described on the K. pneumoniae MLST website $^{1}$. Sequence types (STs) were identified using the online database.

\section{RESULTS AND DISCUSSION}

\section{Antimicrobial Susceptibility}

All CRKP isolates showed multi-resistance to antimicrobials tested. More than 95\% of the CRKP isolates were resistant to 13 antimicrobials tested, with $100 \%$ resistance to piperacillin, piperacillin/tazobactam, ceftazidime, ceftriaxone, cefotaxime, cefoperazone/sulbactam, cefepime, meropenem, and ciprofloxacin. However, the susceptibility rates of these isolates to other antimicrobials tested were high, with susceptibility rates of $95.12 \%$ to tigecycline, $92.68 \%$ to minocycline, $85.37 \%$ to tetracycline, and $85.37 \%$ to trimethoprim-sulfamethoxazole,

\footnotetext{
${ }^{1}$ http://www.pasteur.fr/recherche/genopole/PF8/mlst/Kpneumoniae.html
}

respectively. There was similar resistance pattern between the CRKP isolates from ventilators and patients.

\section{Detection of $\beta$-Lactamases and Resistance Genes}

All CRKP isolates were confirmed as carbapenemase producer determined by the modified Hodge test. In accordance with phenotypic results of carbapenemases, all carbapenemaseproducing isolates were positive for $b l a_{K P C-2}$, with one positive for both bla $a_{K P C-2}$ and bla $a_{N M-1}$ (Figure 1). The result of the present study was similar to previous reports in which bla $a_{K P C-2}$ was the predominant carbapenemase gene among K. pneumoniae in China (Chen et al., 2011; Hu et al., 2012). Other carbapenemase genes including bla $a_{G E S}$, bla $a_{I M P}$, bla $a_{S P M}$, and $b l a_{V I M}$ were not detected in any of the tested isolates. Our previous report also found that 54.9\%, (28/51) of carbapenemresistant Enterobacteriaceae isolates expressed bla $a_{K P C-2}(\mathrm{Hu}$ et al., 2014). In contrast, IMP and NDM type metallo-lactamases were also found among $K$. pneumoniae clinical isolates in China. IMP-4 was found among four K. pneumoniae clinical isolates from neonates and three $K$. pneumoniae isolates from the environment of neonate ICU (Yu et al., 2012). A high prevalence rate $(55.0 \%, 22 / 40)$ of MBLs including IMP-4, IMP8 , and NDM-1 was found among CRKP isolates at a tertiary teaching hospital in southeastern China (Li et al., 2014). Coproduction of different carbapenamases was also found among clinically important organisms which poses a challenge for infection control (Karthikeyan et al., 2010; Wei et al., 2011; Dortet et al., 2012; Kumarasamy and Kalyanasundaram, 2012; Rimrang et al., 2012). In the present study, coexistence of bla $\mathrm{KPC}-2$ and $b l a_{N D M-1}$ was found in one CRKP isolate. More type carbapenemases and co-production of different carbapenamases were found among CPKP isolates in China, which should be of concern.

Co-production of carbapenemases with other $\beta$-lactamases results in resistance to nearly all clinically available $\beta$-lactams. As carbapenemases are not inhibited by clavulanic acid, co-production of ESBLs and carbapenemases can mask identification of ESBLs by the CLSI-recommended doubledisk test. Detection of multiple $\beta$-lactamases produced by Enterobacteriaceae in the clinical laboratory is challenging. In the present study, all carbapenemase-producing isolates harbored at least one of ESBL genes tested, among which blaCTX-M-24 92.7\% (38/41), blaSHV-12 78.1\% (32/41), blaTEM-104 7.3\%, (3/41), and blaCTX-M-3 2.4\% (1/41) harbored (Figure 1). Thirty-seven $(85.37 \%)$ of 41 CRKP isolates were positive for narrow spectrum lactamase gene, $b l_{T E M-1} \cdot 95.1 \%(39 / 41)$ of the tested isolates were found to harbor both $b l a_{C T X}-M-24$ and $b l a_{K P C-2}$, with $75.6 \%$ (31 isolates) with coexistence of $b l a_{C T X-M-24}, b l a_{S H V-12}$, and $b l a_{K P C-2}$ (Figure 1). In particular, two isolates with $b l a_{K P C-2}$ were positive for three ESBL genes including bla $a_{C T X-M-24}, b l a_{S H V-12}$, and bla $a_{T E M-104}$ (Figure 1). Of note, one isolate harbored simultaneously two carbapenemase genes $\left(b l a_{K P C-2}\right.$ and $\left.b l a_{N D M-1}\right)$ and two ESBL genes $\left(b l a_{C T X-M-3}\right.$ and bla $a_{T E M-104}$; Figure 1). bla $a_{K P C-2}$ has been found to coexist with various ESBL genes in one organism. 
Dice (Opt:1.50\%) (Tol 1.0\%-1.0\%) (H>0.0\% S>0.0\%) [0.0\%-100.0\%]

PFGE-Xbal PFGE-Xbal

요, 오, ․․, 요, 운

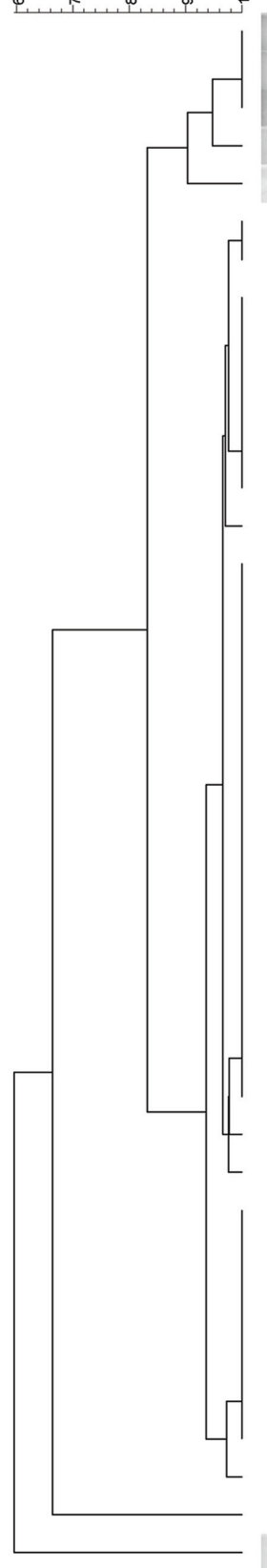

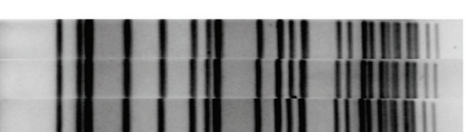
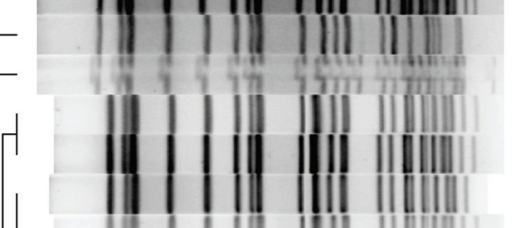

39 sputum

40 BALF3)

41 sputum

38 sputum

36 sputum

15 Blood

19 sputum

3 sputum

13 ascites

14 sputum

20 Ventilator

23 Ventilator

30 Ventilator

7 sputum

4 Ventilator

9 sputum SICU

10 Ventilator

11 sputum SICU

12 Ventilator

17 sputum SICU

18 Ventilator

22 sputum

24 sputum

25 Ventilato

29 sputum

31 sputum

33 urine

34 sputum

35 Sputum

8 Ventilator

16 Ventilator

1 sputum SICU

2 Ventilator

5 sputum SICU

6 Ventilator

26 sputum SICU

27 Ventilator

28 sputum

21 sputum

32 blood

37 bile

SICU
SICU KPC-2

Respiratory KPC-2

KPC-2

Gastroenterology KPC-2

Nephrology KPC-2

SICU KPC-2

SICU KPC-2

SICU KPC-2

general surgery KPC-2

Neurosurgery KPC-2

KPC-2

KPC-2

KPC-2

KPC-2

KPC-2

KPC-2

KPC-2

KPC-2

KPC-2

KPC-2

KPC-2

KPC-2

KPC-2

KPC-2

KPC-2

KPC-2

KPC-2

KPC-2

KPC-2

KPC-2

KPC-2

KPC-2

KPC-2

KPC-2

KPC-2

KPC-2

KPC-2

KPC-2

KPC-2

KPC-2, NDM-1 1855 CTX-M-3, TEM-104

Gastroenterology KPC-2

11 SHV-12,CTX-M-24
11 SHV-12,CTX-M-24

11 SHV-12,CTX-M-24

11 SHV-12,CTX-M-24

11 SHV-12,CTX-M-24

11 CTX-M-24

11 SHV-12,CTX-M-24

11 SHV-12,CTX-M-24

11 CTX-M-24

11 CTX-M-24

11 SHV-12

11 CTX-M-24

11 SHV-12,CTX-M-24

11 SHV-12,CTX-M-24

11 SHV-12,CTX-M-24

11 SHV-12,CTX-M-24

11 SHV-12,CTX-M-24

11 SHV-12,CTX-M-24, TEM-104

11 SHV-12,CTX-M-24

11 CTX-M-24

11 CTX-M-24

11 SHV-12,CTX-M-24

11 SHV-12,CTX-M-24

11 SHV-12,CTX-M-24

11 SHV-12,CTX-M-24

11 SHV-12,CTX-M-24

11 SHV-12,CTX-M-14

11 SHV-12,CTX-M-24

11 SHV-12,CTX-M-24

11 SHV-12,CTX-M-24

11 SHV-12,CTX-M-24

11 SHV-12,CTX-M-24,TEM-104

11 SHV-12,CTX-M-24

11 SHV-12,CTX-M-24

11 SHV-12,CTX-M-24

11 SHV-12,CTX-M-24

11 SHV-12,CTX-M-24

11 SHV-12,CTX-M-24

1854 CTX-M-24

1224 CTX-M-24

FIGURE 1 | Dendrogram of patterns for carbapenem-resistant $K$. pneumonia isolates obtained by PFGE. SICU, surgical intensive care unit; BALF, bronchoalveolar lavage fluid; ST, sequence type; ESBLs, extended-spectrum beta-lactamases.

However, to the best of our knowledge, coexistence of bla $a_{K P C}-2$ and bla $C T X-M-24$ in one organism is first reported.

\section{Bacterial Clonal Relatedness}

Multi-locus sequence typing results showed that 41 CRKP isolates belonged to four STs including ST11, novel ST1854, novel ST855, and ST1224 (Figure 1). PFGE results displayed three PFGE clusters (Figure 1). Thirty-eight isolates (92.7\%) including all 13 isolates from ventilators and 25 isolates from patients from six wards belonged to ST11. In accordance with MLST results, PFGE results also showed that 38 ST11 isolates belonged to same PFGE cluster with more than $80 \%$ of similarity, indicating these CRKP isolates were clonally related. Particularly, 15 isolates have an identical undistinguished pattern (100\% similarity) forming 
a single clonal population. Moreover, this clone was exclusively linked to the cases attended in SICU and linked to the ventilators. Additionally, the other SICU cases were linked to closely related clones (similarity greater than 95\%). These data indicated that the occurrence of a clonal outbreak associated with ventilators has been found.

Although the isolate harboring both $b l a_{K P C-2}$ and $b l a_{N D M-1}$ was also from surgery ICU, it belonged to a novel ST, ST1855, which was a two-locus variant of ST11. The ST1855 isolate belonged to a specific PFGE cluster different from the PFGE cluster representing for ST11 isolates, indicating that the ST1855 isolate was not clonally related to ST11 isolates. Another novel ST, ST1854, was found in a isolate from the sputum of a patient in respiratory ward. As ST1854 was a single-locus variant of ST11, the ST1854 isolate and ST11 isolates belonged to same PFGE cluster. The ST1224 isolate belonged to another PFGE cluster. Among 25 patients infected by ST11 isolates, 18 were from surgery ICU and other seven were from five wards. ST11, a single-locus variant of ST258, was demonstrated to be the predominant ST among KPC-2 producing K. pneumoniae in China (Li et al., 2012). ST11 was also prevalent in Brazil (Andrade et al., 2014). Although ST258 is the epidemic clone of KPC-2 producing $K$. pneumoniae worldwide, which has been reported as dominant clone in USA (Kitchel et al., 2009), this important clone has not been detected in China. Our data indicated that the outbreak by KPC-2 -producing K. pneumonia has been found in SICU of this tertiary teaching hospital in central China. Other than surgery ICU, sparse ST11 isolates belonging to same PFGE cluster were found in general surgery ward, Neurosurgery ward, Urology ward, and Nephrology ward, indicating KPC2 -producing ST11 K. pneumoniae clone has disseminated at this hospital. It is of concern that outbreak by this important clone will occurred in other wards. Pulmonary infection caused by ST11 CRKP was found among18 patients from surgery ICU who were submitted to mechanical ventilation using the ventilators contaminated by KPC-2 -producing $K$. pneumoniae. Moreover, the CRKP isolates from patients from surgery ICU were clonally related to the isolates from ventilators. Therefore,

\section{REFERENCES}

Andrade, L. N., Minarini, L. A., Pitondo-Silva, A., Climaco, E. C., Palazzo, I. C., Medeiros, M. I., et al. (2010). Determinants of beta-lactam resistance in meningitis-causing Enterobacteriaceae in Brazil. Can. J. Microbiol. 56, 399-407. doi: 10.1139/w10-020

Andrade, L. N., Vitali, L., Gaspar, G. G., Bellissimo-Rodrigues, F., Martinez, R., and Darini, A. L. (2014). Expansion and evolution of a virulent, extensively drugresistant (polymyxin B-resistant), QnrS1-, CTX-M-2-, and KPC-2-producing Klebsiella pneumoniae ST11 international high-risk clone. J. Clin. Microbiol. 52, 2530-2535. doi: 10.1128/JCM.00088-14

Bratu, S., Landman, D., Haag, R., Recco, R., Eramo, A., Alam, M., et al. (2005). Rapid spread of carbapenem-resistant Klebsiella pneumoniae in New York City: a new threat to our antibiotic armamentarium. Arch. Intern. Med. 165, 1430-1435. doi: 10.1001/archinte.165.12.1430

Cai, J. C., Zhou, H. W., Zhang, R., and Chen, G. X. (2008). Emergence of Serratia marcescens, Klebsiella pneumoniae, and Escherichia coli isolates possessing the plasmid-mediated carbapenem-hydrolyzing beta-lactamase KPC-2 in intensive care units of a Chinese hospital. Antimicrob. Agents Chemother. 52, 2014-2018. doi: 10.1128/AAC.01539-07 we speculated that the outbreak by KPC-2 -producing ST11 K. pneumoniae clone was associated with ventilators. To control the spread of carbapenemase-producing organism in hospital, strict infection control measures such as reinforcement of hand hygiene, contact precautions, disinfection of equipments, disinfection of contaminated environment around patients should be carried out.

\section{CONCLUSION}

An outbreak caused by ventilator-associated ST11 K. pneumoniae with co-production of CTX-M-24 and KPC-2 has been found in a surgery ICU of a tertiary teaching hospital in central China.

\section{AUTHOR CONTRIBUTIONS}

LH, YL, LD, QZ, YH, ZW, and LZ performed the laboratory measurements. FY and LW made substantial contributions to conception and design. FY and LW revised the manuscript critically for important intellectual content. YL, LH, and FY participated in experimental design and data analysis. FY drafted the manuscript. All authors read and approved the final manuscript.

\section{FUNDING}

This study was supported by research grant (20112BBG70054) by Jiangxi provincial department of science and technology, China.

\section{ACKNOWLEDGMENT}

We thank the staff from clinical microbiology laboratory, department of clinical laboratory, Jiangxi Provincial People's Hospital, for kindly providing CRKP isolates.
Chen, S., Hu, F., Xu, X., Liu, Y., Wu, W., Zhu, D., et al. (2011). High prevalence of KPC-2-type carbapenemase coupled with CTX-M-type extendedspectrum beta-lactamases in carbapenem-resistant Klebsiella pneumoniae in a teaching hospital in China. Antimicrob. Agents Chemother. 55, 2493-2494. doi: 10.1128/AAC.00047-11

Clinical and Laboratory Standards Institute [CLSI] (2014). Performance Standards for Antimicrobial Susceptibility Testing, 24th Informational Supplement (M100S24). Wayne, PA: Clinical and Laboratory Standards Institute.

Dortet, L., Poirel, L., Al Yaqoubi, F., and Nordmann, P. (2012). NDM1, OXA-48 and OXA-181 carbapenemase-producing Enterobacteriaceae in Sultanate of Oman. Clin. Microbiol. Infect. 18, E144-E148. doi: 10.1111/j.14690691.2012.03796.x

Gupta, N., Limbago, B. M., Patel, J. B., and Kallen, A. J. (2011). Carbapenemresistant Enterobacteriaceae: epidemiology and prevention. Clin. Infect. Dis. 53, 60-67. doi: 10.1093/cid/cir202

Hammerum, A. M., Hansen, F., Lester, C. H., Jensen, K. T., Hansen, D. S., and Dessau, R. B. (2010). Detection of the first two Klebsiella pneumoniae isolates with sequence type 258 producing KPC-2 carbapenemase in Denmark. Int. J. Antimicrob. Agents 35, 610-612. doi: 10.1016/j.ijantimicag.2010. 01.024 
Hu, F., Chen, S., Xu, X., Guo, Y., Liu, Y., Zhu, D., et al. (2012). Emergence of carbapenem-resistant clinical Enterobacteriaceae isolates from a teaching hospital in Shanghai, China. J. Med. Microbiol. 61, 132-136. doi: 10.1099/jmm.0.036483-0

Hu, L., Zhong, Q., Shang, Y., Wang, H., Ning, C., Li, Y., et al. (2014). The prevalence of carbapenemase genes and plasmid-mediated quinolone resistance determinants in carbapenem-resistant Enterobacteriaceae from five teaching hospitals in central China. Epidemiol. Infect. 142, 1972-1977. doi: 10.1017/S0950268813002975

Karthikeyan, K., Thirunarayan, M. A., and Krishnan, P. (2010). Coexistence of blaOXA-23 with blaNDM-1 and armA in clinical isolates of Acinetobacter baumannii from India. J. Antimicrob. Chemother. 65, 2253-2254. doi: 10.1093/jac/dkq273

Kitchel, B., Rasheed, J. K., Patel, J. B., Srinivasan, A., Navon-Venezia, S., Carmeli, Y., et al. (2009). Molecular epidemiology of KPC-producing Klebsiella pneumoniae isolates in the United States: clonal expansion of multilocus sequence type 258. Antimicrob. Agents Chemother. 53, 3365-3370. doi: 10.1128/AAC.00126-09

Kumarasamy, K., and Kalyanasundaram, A. (2012). Emergence of Klebsiella pneumoniae isolate co-producing NDM-1 with KPC-2 from India. J. Antimicrob. Chemother. 67, 243-244. doi: 10.1093/jac/ dkr431

Kumarasamy, K. K., Toleman, M. A., Walsh, T. R., Bagaria, J., Butt, F., Balakrishnan, R., et al. (2010). Emergence of a new antibiotic resistance mechanism in India, Pakistan, and the UK: a molecular, biological, and epidemiological study. Lancet Infect. Dis. 10, 597-602. doi: 10.1016/S14733099(10)70143-2

Li, B., Xu, X. H., Zhao, Z. C., Wang, M. H., and Cao, Y. P. (2014). High prevalence of metallo-beta-lactamase among carbapenem-resistant Klebsiella pneumoniae in a teaching hospital in China. Can. J. Microbiol. 60, 691-695. doi: 10.1139/cjm-2014-0291

Li, J. J., Sheng, Z. K., Deng, M., Bi, S., Hu, F. S., Miao, H. F., et al. (2012). Epidemic of Klebsiella pneumoniae ST11 clone coproducing KPC-2 and 16S rRNA methylase RmtB in a Chinese University Hospital. BMC Infect. Dis. 12:373. doi: 10.1186/1471-2334-12-373

Marquez, C., Ingold, A., Echeverria, N., Acevedo, A., Vignoli, R., Garcia-Fulgueiras, V., et al. (2014). Emergence of KPC-producing Klebsiella pneumoniae in Uruguay: infection control and molecular characterization. New Microbes New Infect. 2, 58-63. doi: 10.1002/ nmi2.40

Nordmann, P., and Poirel, L. (2002). Emerging carbapenemases in Gramnegative aerobes. Clin. Microbiol. Infect. 8, 321-331. doi: 10.1046/j.14690691.2002.00401.x

Nordmann, P., Poirel, L., Carrer, A., Toleman, M. A., and Walsh, T. R. (2011). How to detect NDM-1 producers. J. Clin. Microbiol. 49, 718-721. doi: 10.1128/JCM.01773-10
Qi, Y., Wei, Z., Ji, S., Du, X., Shen, P., and Yu, Y. (2011). ST11, the dominant clone of KPC-producing Klebsiella pneumoniae in China. J Antimicrob Chemother. 66, 307-312. doi: 10.1093/jac/dkq431

Queenan, A. M., and Bush, K. (2007). Carbapenemases: the versatile betalactamases. Clin. Microbiol. Rev. 20, 440-458. doi: 10.1128/CMR.00001-07

Rimrang, B., Chanawong, A., Lulitanond, A., Wilailuckana, C., Charoensri, N., Sribenjalux, P., et al. (2012). Emergence of NDM-1- and IMP-14a-producing Enterobacteriaceae in Thailand. J. Antimicrob. Chemother. 67, 2626-2630. doi: $10.1093 / \mathrm{jac} / \mathrm{dks} 267$

Temkin, E., Adler, A., Lerner, A., and Carmeli, Y. (2014). Carbapenem-resistant Enterobacteriaceae: biology, epidemiology, and management. Ann. N. Y. Acad. Sci. 1323, 22-42. doi: 10.1111/nyas. 12537

Wei, Z., Yu, T., Qi, Y., Ji, S., Shen, P., Yu, Y., et al. (2011). Coexistence of plasmid-mediated KPC-2 and IMP-4 carbapenemases in isolates of Klebsiella pneumoniae from China. J. Antimicrob. Chemother. 66, 2670-2671. doi: $10.1093 / \mathrm{jac} / \mathrm{dkr} 330$

Wu, Q., Liu, Q., Han, L., Sun, J., and Ni, Y. (2010). Plasmid-mediated carbapenem-hydrolyzing enzyme KPC-2 and ArmA 16S rRNA methylase conferring high-level aminoglycoside resistance in carbapenem-resistant Enterobacter cloacae in China. Diagn. Microbiol. Infect. Dis. 66, 326-328. doi: 10.1016/j.diagmicrobio.2009.10.003

Yang, J., Ye, L., Guo, L., Zhao, Q., Chen, R., Luo, Y., et al. (2013). A nosocomial outbreak of KPC-2-producing Klebsiella pneumoniae in a Chinese hospital: dissemination of ST11 and emergence of ST37, ST392 and ST395. Clin. Microbiol. Infect. 19, E509-E515. doi: 10.1111/1469-0691.12275

Yong, D., Toleman, M. A., Giske, C. G., Cho, H. S., Sundman, K., Lee, K., et al. (2009). Characterization of a new metallo-beta-lactamase gene, bla(NDM-1), and a novel erythromycin esterase gene carried on a unique genetic structure in Klebsiella pneumoniae sequence type 14 from India. Antimicrob. Agents Chemother. 53, 5046-5054. doi: 10.1128/AAC.00774-09

Yu, F., Ying, Q., Chen, C., Li, T., Ding, B., Liu, Y., et al. (2012). Outbreak of pulmonary infection caused by Klebsiella pneumoniae isolates harbouring blaIMP-4 and blaDHA-1 in a neonatal intensive care unit in China. J. Med. Microbiol. 61, 984-989. doi: 10.1099/jmm.0.043000-0

Conflict of Interest Statement: The authors declare that the research was conducted in the absence of any commercial or financial relationships that could be construed as a potential conflict of interest.

Copyright (c) 2016 Hu, Liu, Deng, Zhong, Hang, Wang, Zhan, Wang and Yu. This is an open-access article distributed under the terms of the Creative Commons Attribution License (CC BY). The use, distribution or reproduction in other forums is permitted, provided the original author(s) or licensor are credited and that the original publication in this journal is cited, in accordance with accepted academic practice. No use, distribution or reproduction is permitted which does not comply with these terms. 